\title{
Improvement of sugarcane (Saccharum officinarum L. var. Isd. 39) using gamma irradiation and large scale plantlet production from $M_{1}$ generation through in vitro culture
}

\author{
Shamima Akter*, N. Alam and P.K. Roy ${ }^{1}$ \\ Department of Botany, Jahangirnagar University, Savar, Dhaka, Bangladesh
}

\begin{abstract}
The present study was aimed to develop mutant sugarcane (Saccharum officinarum L. var. Isd. 39) using gamma radiation followed by regeneration of those mutants through in vitro technique. Five different doses of gamma radiation (10, 20, 30, 40 and $50 \mathrm{~Gy}$ ) were applied to the sugarcane setts and among these doses $10 \mathrm{~Gy}$ irradiated mutant sugarcane showed the best agronomical traits. In addition, $10 \mathrm{~Gy}$ irradiated mutant sugarcane also contained higher sugar concentrations (14.25\%) compared to control (14.23\%). On the other hand, shoot tip and young folded leaf segments from the $10 \mathrm{~Gy}$ irradiated plants $\left(\mathrm{M}_{1}\right.$ generation) were used as explants in in vitro culture. It was evident from the experiment that direct shoot regeneration was better than indirect regeneration. Young leaf segments produced the highest percentage $(84 \%)$ of shoots when cultured on MS+1.0 mg/l Kin $+0.2 \mathrm{mg} / 1 \mathrm{NAA}$. The mean number of shoots was recorded as $35 \pm 1.12$ in the same medium. Moreover, addition of $4 \%$ sucrose and $10 \%$ coconut water $(\mathrm{CW})$ in the same medium increased the number of shoot up to 45 per culture. Best rooting was obtained from shoots cultured on half-strength of MS fortified with $1.0 \mathrm{mg} / 1 \mathrm{NAA}$ and $0.5 \mathrm{mg} / \mathrm{l} \mathrm{IBA}$. Well-rooted plantlets were transferred to poly bag containing soil and compost (2:1) for hardening. During hardening 70\% plantlets survived, which were subsequently transferred to the experimental field.
\end{abstract}

Key words: Saccharum officinarum L. var. Isd. 39, In vitro culture, Explants, Regeneration.

\section{INTRODUCTION}

Sugarcane is a tall and relatively strong class of perennial grasses that are known to have high sugar content. Commonly known sugarcane species scientific name is Saccharum officinarum which belongs to the family Poaceae. It is one of the most important industrial crops in both the tropical and subtropical regions of the world and is the principal raw material for the sugar industry (Khan et al., 2004). The use of gamma radiation to induce mutation is a method that has been applied in plant breeding to increase genetic variations (Brunner, 1995). It has also been used as an effective method, which can greatly induce high mutation numbers and modify physiological characteristics to create new mutants with improved properties (Ahloowalia and Maluszynski, 2001, Bermejo et al., 2011, Predieri, 2001). True genetic changes are desirable in mutation studies. Many fruitful agronomical changes (high cane yield, high sucrose content) were recorded in the treated material (Anonymous, 1953). In addition,

\footnotetext{
${ }^{1}$ Plant Biotechnology and Genetic Engineering Division, IFRB, Atomic Energy Research Establishment, Savar, Dhaka, Bangladesh

*Corresponding author. E-mail: shamima.akter221@gmail.com
} 
tissue culture as a tool of advanced plant breeding when coupled with mutation breeding would become an important and valuable tool in the hands of plant breeders to create genetic variability for the selection of new genotypes with improved agronomic characteristics.

On the other hand, micropropagation is currently the only realistic means of achieving rapid, large-scale production of disease-free quality planting material as seed canes of newly developed varieties in order to speed up the breeding and commercialization process in Sugarcane (Feldmennet al.,1994; Lal \& Krishna,1994; Lee , 1987; Lorenzo et al., 2001; Krisnamurthi and Tlaskal, 1974). As a result of which plant regeneration through tissue culture technique would be a viable alternative for improving the quality and productivity in sugarcane.

Sugarcane (Saccharum officinarum L. var. Isd. 39) is one of the important agricultural cash crops in Bangladesh and is the major source of sugar. Unfortunately, scientific information about the application of mutation breeding by gamma radiation to improve mutant and from which in vitro tissue culture for large scale production is very much scanty or absent. So, the present study was conducted to improve a sugarcane variety (Saccharum officinarum L. var. Isd. 39) by gamma irradiation and to establish a protocol for large scale plantlets production of that mutant through in vitro culture.

\section{MATERIALS AND METHODS}

The investigation was performed in two phases. First phase was gamma radiation treatment in a sugarcane variety for induction of mutation and the second phase was in vitro propagation of the superior mutant.

High yielding variety of sugarcane (Saccharum officinarum L.) namely Isd. 39 were collected from Bangladesh Sugarcane Research Institute (BSRI), Ishwardi, Pabna. About 900 cane setts containing one eyebud were used as plant material. About 900 cane setts were prepared from collected sugarcane. Thereafter, total cane setts were divided into six batches. First batch was treated as control and rest of the batches like 2, 3, 4, 5 and 6 were subjected to $10,20,30,40$ and 50 Gy gamma radiations respectively in a 50,000-curie panoramic ${ }^{60} \mathrm{Co}$ source in a radiation chamber of IFRB, AERE. The irradiated cane setts were then transferred to the pre prepared experimental plots. Various agronomical traits namely plant height, nodal diameter and internodal length were recorded after 1.5, 2.5, 4, $5,7,10$ and 12 months of interval.

In vitro propagation of superior $\mathrm{M}_{1}$ generation was adopted in order to maintain the characteristic of it. The germinated plants are $\mathrm{M}_{1}$ progeny as the seed materials were exposed by induced mutagen (gamma ray). Shoot tips and young folded leaves of $\mathbf{M}_{1}$ progeny (selected elite mother plant) were collected from the experimental field and were used as explants. In the present investigation MS and half strength MS media supplemented with different concentrations and combinations of plant growth regulators (PGRs) were used for the purpose of shoot proliferation and root induction. Surface sterilization of explants (shoot tip and young folded leaves) was carried out by $0.1 \%$ $\mathrm{HgCl}_{2}$ solution for 6 minutes. Thereafter explants cut into small pieces and inoculated in 
the culture vessels containing nutrient medium. The culture vessels containing explants were incubated in a growth chamber. The temperature of the growth chamber was $25 \pm 2^{\circ} \mathrm{C}, 16$ hours photoperiod and 3000 lux (approx.) photo intensity. Subculture for shoot multiplication and root induction were done at a regular interval of four weeks. The regenerated healthy plantlets after developing sufficient root system were transferred to the soil. The percentage $(\%)$ of contamination free explants, the responses of different explants towards shoot regeneration and rooting were recorded carefully. Duncan's multiple-range test (DMRT) was carried out at 5\% probability level according to Federer (1963).

\section{RESULTS AND DISCUSSIONS}

In the present study, regarding the agronomical traits the percentage of germination was highest $(70 \%)$ in controlled plants and the second highest percentage was in 10 Gy (48\%) irradiated plants among all the irradiated plants. The other irradiated plants i.e., 20, 3040 and $50 \mathrm{~Gy}$ showed the rate of germination as $42 \%, 36 \%, 30 \%$ and $24 \%$ respectively. The lowest percentage $(24 \%)$ was observed in 50 Gy irradiated plants. According to KwonNdung and Ifenkwe (2000), 4 to 8 KGy were identified for optimal germination of cane buds and higher doses decrease the percentage of germination. Higher doses of gamma rays delay and suppress germination of sugarcane buds. Nwachukwu et al. (1990) observed similar behavior with sprouting of gamma treated yam micro-tubers. The present results confirm these earlier reports in soybean (Pepol and Pepo 1989 and Pavadai et al., 2009); mung bean and chickpea (Khan et al., 2009 and Khan \& Wani, 2005) and sesame (Prabhakar, 1985). Yasmin et al. (2011) observed maximum and minimum plant regeneration in 20 Gy and 40 Gy irradiation respectively.

Among all the irradiation, 10 Gy showed best performance in plant height (Table 1). Khan et al. (2007) observed that 20 Gy irradiated mutant showed best performance in plant height. This observation was more or less similar with the present findings. In the present study the highest nodal diameter recorded after five, seven, ten and twelve months were $4.1 \mathrm{~cm}, 5.2 \mathrm{~cm}, 7.3 \mathrm{~cm}$ and $7.5 \mathrm{~cm}$ respectively in $10 \mathrm{~Gy}$ irradiated plants whereas, the lowest nodal diameter was in 50 Gy irradiated plants (Table 1). Again, the 10 Gy irradiated plants showed the best internodal length among all the plants which were 2.2 $\mathrm{cm}, 3.5 \mathrm{~cm}, 8.1 \mathrm{~cm}$ and $8.2 \mathrm{~cm}$ after five, seven, ten and twelve month of sowing respectively. Plant height and plant girth are the main contributing traits in determining cane yield (Rehman et al., 1992; Khan et al., 1997). In addition, 10 Gy irradiated mutant sugarcane also showed somewhat highest sugar content $(14.25 \%)$ compare to others (Table 1).

On the other hand, present investigation was undertaken to establish a protocol of in vitro plant regeneration from shoot tip and young folded leaf segment explants of improved $\mathrm{M}_{1}$ line. Total experiment was performed in three phases. In first phase direct regeneration of plants was tried with shoot tip and young folded leaf segments. In second phase, indirect regeneration was tried to establish from young folded leaf segment derived callus. Finally in the third phase rooting, hardening and establishment of plantlets to experimental field was done. 
Table 1. Agronomical characteristics of the mutants and controlled sugarcane plants during the study periods

\begin{tabular}{|c|c|c|c|c|c|c|c|c|c|c|c|c|c|c|c|c|c|c|c|c|c|c|c|c|}
\hline \multirow{2}{*}{$\begin{array}{l}\text { Mo } \\
\text { nth }\end{array}$} & \multicolumn{6}{|c|}{ Plant height $(\mathrm{cm})$} & \multicolumn{6}{|c|}{ Nodal diameter $(\mathrm{cm})$} & \multicolumn{6}{|c|}{ Internodal length $(\mathrm{cm})$} & \multicolumn{6}{|c|}{ Sugar content (\%) } \\
\hline & $\begin{array}{l}\text { Co } \\
\text { nt. }\end{array}$ & $\begin{array}{l}10 \\
\text { Gy }\end{array}$ & $\begin{array}{l}20 \\
\text { Gy }\end{array}$ & $\begin{array}{l}30 \\
\text { Gy }\end{array}$ & $\begin{array}{l}40 \\
\text { Gy }\end{array}$ & $\begin{array}{l}50 \\
\text { Gy }\end{array}$ & $\begin{array}{l}\text { Co } \\
\text { nt. }\end{array}$ & $\begin{array}{l}10 \\
\text { Gy }\end{array}$ & $\begin{array}{l}20 \\
\text { Gy }\end{array}$ & $\begin{array}{l}30 \\
\text { Gy }\end{array}$ & $\begin{array}{l}40 \\
\text { Gy }\end{array}$ & $\begin{array}{l}50 \\
\text { Gy }\end{array}$ & $\begin{array}{l}\text { Co } \\
\text { nt. }\end{array}$ & $\begin{array}{l}10 \\
\text { Gy }\end{array}$ & $\begin{array}{l}20 \\
\text { Gy }\end{array}$ & $\begin{array}{l}30 \\
\text { Gy }\end{array}$ & $\begin{array}{l}40 \\
\text { Gy }\end{array}$ & $\begin{array}{l}50 \\
\text { Gy }\end{array}$ & $\begin{array}{l}\text { Co } \\
\text { nt. }\end{array}$ & $\begin{array}{l}10 \\
\text { Gy }\end{array}$ & $\begin{array}{l}20 \\
\text { Gy }\end{array}$ & $\begin{array}{l}30 \\
\text { Gy }\end{array}$ & $\begin{array}{l}40 \\
\text { Gy }\end{array}$ & $\begin{array}{l}50 \\
\text { Gy }\end{array}$ \\
\hline 1.5 & $\begin{array}{l}11 . \\
25 a\end{array}$ & $\begin{array}{l}14 . \\
06 a\end{array}$ & $\begin{array}{l}13 . \\
25 a\end{array}$ & $\begin{array}{l}11 . \\
20 \mathrm{a}\end{array}$ & $\begin{array}{l}10 . \\
06 a\end{array}$ & $\begin{array}{l}9.6 \\
0 \mathrm{a}\end{array}$ & - & - & - & - & - & - & - & - & - & - & - & - & - & - & - & - & - & \\
\hline 2.5 & $\begin{array}{l}12 . \\
75 a\end{array}$ & $21 \mathrm{c}$ & $\begin{array}{l}15 \\
31 \\
\text { b }\end{array}$ & $\begin{array}{l}12 . \\
62 \mathrm{a}\end{array}$ & $\begin{array}{l}11 . \\
75 a\end{array}$ & $\begin{array}{l}11 . \\
2 a\end{array}$ & - & - & - & - & - & - & - & - & - & - & - & - & - & - & - & - & - & \\
\hline 4 & $\begin{array}{l}28 \\
b\end{array}$ & $\begin{array}{l}47 . \\
8 c\end{array}$ & $\begin{array}{l}38 . \\
87 \\
\text { d }\end{array}$ & $\begin{array}{l}31 . \\
66 c\end{array}$ & $18 \mathrm{a}$ & $\begin{array}{l}16 . \\
5 a\end{array}$ & - & - & - & - & - & - & - & - & - & - & - & - & - & - & - & - & - & \\
\hline 5 & - & - & - & - & - & - & $\begin{array}{l}3.5 \\
a b\end{array}$ & $\begin{array}{l}4.1 \\
b\end{array}$ & $\begin{array}{l}3.6 \\
\mathrm{a}\end{array}$ & $\begin{array}{l}3.2 \\
\mathrm{a}\end{array}$ & $\begin{array}{l}3.2 \\
\mathrm{a}\end{array}$ & $\begin{array}{l}3.1 \\
a b\end{array}$ & $2 b c$ & $\begin{array}{l}2.2 \\
d\end{array}$ & $\begin{array}{l}2.1 \\
\mathrm{~cd}\end{array}$ & $2 b c$ & $\begin{array}{l}1.9 \\
a b\end{array}$ & $\begin{array}{l}1.8 \\
\mathrm{a}\end{array}$ & - & - & - & - & - & \\
\hline 7 & $\begin{array}{l}16 \\
0 \mathrm{~cd}\end{array}$ & $\begin{array}{l}16 \\
5 \mathrm{~d}\end{array}$ & $\begin{array}{l}15 \\
8 \mathrm{c}\end{array}$ & $\begin{array}{l}15 \\
0 b\end{array}$ & $\begin{array}{l}14 \\
8 b\end{array}$ & $\begin{array}{l}140 \\
\mathrm{a}\end{array}$ & $\begin{array}{l}4.8 \\
\mathrm{~b}\end{array}$ & $\begin{array}{l}5.2 \\
b\end{array}$ & $\begin{array}{l}4.3 \\
\mathrm{a}\end{array}$ & $\begin{array}{l}4.2 \\
\mathrm{a}\end{array}$ & $\begin{array}{l}4.3 \\
\mathrm{a}\end{array}$ & $\begin{array}{l}3.8 \\
\mathrm{a}\end{array}$ & $\begin{array}{l}3.1 \\
\mathrm{a}\end{array}$ & $\begin{array}{l}3.5 \\
b\end{array}$ & $\begin{array}{l}2.9 \\
\mathrm{a}\end{array}$ & $3 a$ & $\begin{array}{l}2.8 \\
\mathrm{a}\end{array}$ & $\begin{array}{l}2.9 \\
\mathrm{a}\end{array}$ & - & - & - & - & - & \\
\hline 10 & $\begin{array}{l}26 \\
1 \mathrm{~cd}\end{array}$ & $\begin{array}{l}27 \\
0 \mathrm{~d}\end{array}$ & $\begin{array}{l}25 \\
7 \mathrm{c}\end{array}$ & $\begin{array}{l}25 \\
2 b\end{array}$ & $\begin{array}{l}24 \\
7 b\end{array}$ & $\begin{array}{l}241 \\
\mathrm{a}\end{array}$ & $\begin{array}{l}6.9 \\
c\end{array}$ & $\begin{array}{l}7.3 \\
d\end{array}$ & $\begin{array}{l}6.4 \\
b\end{array}$ & $\begin{array}{l}6.2 \\
a b\end{array}$ & $\begin{array}{l}6.2 \\
a b\end{array}$ & $\begin{array}{l}5.9 \\
\mathrm{a}\end{array}$ & $\begin{array}{l}7.3 \\
\text { b }\end{array}$ & $\begin{array}{l}8.1 \\
\mathrm{c}\end{array}$ & $\begin{array}{l}7.2 \\
b\end{array}$ & $\begin{array}{l}6.8 \\
\mathrm{a}\end{array}$ & $\begin{array}{l}6.9 \\
\mathrm{a}\end{array}$ & $\begin{array}{l}6.7 \\
\mathrm{a}\end{array}$ & - & - & - & - & - & \\
\hline 12 & $\begin{array}{l}36 \\
5 \mathrm{bc}\end{array}$ & $\begin{array}{l}37 \\
5 c\end{array}$ & $\begin{array}{l}36 \\
1 b\end{array}$ & $\begin{array}{l}35 \\
5 \mathrm{ab}\end{array}$ & $\begin{array}{l}35 \\
2 \mathrm{ab}\end{array}$ & $\begin{array}{l}345 \\
\mathrm{a}\end{array}$ & $7 \mathrm{~d}$ & $\begin{array}{l}7.5 \\
\mathrm{e}\end{array}$ & $\begin{array}{l}6.6 \\
\mathrm{c}\end{array}$ & $\begin{array}{l}6.3 \\
b\end{array}$ & $\begin{array}{l}6.2 \\
a b\end{array}$ & $6 a$ & $\begin{array}{l}7.4 \\
\text { b }\end{array}$ & $\begin{array}{l}8.2 \\
c\end{array}$ & $\begin{array}{l}7.3 \\
b\end{array}$ & $\begin{array}{l}6.9 \\
a\end{array}$ & $\begin{array}{l}6.9 \\
\mathrm{a}\end{array}$ & $\begin{array}{l}6.8 \\
\mathrm{a}\end{array}$ & $\begin{array}{l}14 . \\
23\end{array}$ & $\begin{array}{l}14 . \\
25\end{array}$ & $\begin{array}{l}14 . \\
23\end{array}$ & $\begin{array}{l}14 . \\
22\end{array}$ & $\begin{array}{l}14 . \\
22\end{array}$ & $\begin{array}{l}14 . \\
21\end{array}$ \\
\hline
\end{tabular}

*Mean values followed by the same letter in the same column are not significantly different based on DMRT $(\mathrm{P}=0.05)$. 
Table 2. Effects of different concentrations and combinations of PGRs in MS on shoot regeneration of Saccharum officinarum $L$. var. Isd. 39 using shoot tip and young folded leaf segment from $10 \mathrm{~Gy}$ irradiated $M_{1}$ generation

\begin{tabular}{|c|c|c|c|c|}
\hline \multirow{3}{*}{$\begin{array}{l}\text { Growth regulators } \\
\qquad\left(\mathrm{mgl}^{-1}\right)\end{array}$} & \multicolumn{4}{|c|}{ Explants } \\
\hline & \multicolumn{2}{|c|}{ Shoot tip } & \multicolumn{2}{|c|}{ Young folded leaf } \\
\hline & $\begin{array}{l}\% \text { of explants } \\
\text { producing } \\
\text { shoots }\end{array}$ & $\begin{array}{l}\text { Mean number of } \\
\text { shoots per culture } \\
( \pm \mathrm{SE})\end{array}$ & $\begin{array}{l}\% \text { of explants } \\
\text { producing } \\
\text { shoots }\end{array}$ & $\begin{array}{c}\text { Mean number of } \\
\text { shoots per } \\
\text { culture }( \pm \mathrm{SE})\end{array}$ \\
\hline \multicolumn{5}{|l|}{ BAP (mg/l) } \\
\hline 1.2 & $20 \mathrm{c}$ & $2 \pm 0.09 b c$ & $30 \mathrm{c}$ & $5 \pm 0.09 c$ \\
\hline 1.5 & $26 \mathrm{~d}$ & $3 \pm 0.25 \mathrm{~cd}$ & $40 \mathrm{c}$ & $9 \pm 0.25 d$ \\
\hline 2.0 & $60 f$ & $6 \pm 1.33 e$ & 64d & $12 \pm 1.35 \mathrm{e}$ \\
\hline 2.2 & $42 \mathrm{e}$ & $4 \pm 1.12 d$ & $44 \mathrm{c}$ & $5 \pm 1.08 c$ \\
\hline 2.5 & $28 \mathrm{~d}$ & $3 \pm 0.42 \mathrm{~cd}$ & $20 \mathrm{c}$ & $4 \pm 0.25 c$ \\
\hline \multicolumn{5}{|l|}{ Kin (mg/l) } \\
\hline 1.0 & $12 b$ & $4 \pm 0.08 b c$ & $20 d$ & $5 \pm 1.45 b$ \\
\hline 1.2 & $30 d$ & $5 \pm 0.09 c$ & $28 \mathrm{e}$ & $9 \pm 1.34 \mathrm{c}$ \\
\hline 1.5 & $50 \mathrm{e}$ & $9 \pm 1.25 \mathrm{~d}$ & $15 c$ & $10 \pm 1.09 \mathrm{~cd}$ \\
\hline 2.0 & $68 f$ & $13 \pm 1.32 \mathrm{e}$ & $70 f$ & $12 \pm 1.12 \mathrm{~d}$ \\
\hline 2.2 & $22 c$ & $3 \pm 0.24 b c$ & $18 \mathrm{~cd}$ & $5 \pm 1.08 b$ \\
\hline 3.0 & $10 \mathrm{~b}$ & $2 \pm 0.12 \mathrm{ab}$ & $8 b$ & $5 \pm 1.13 b$ \\
\hline \multicolumn{5}{|l|}{ Zeatin (mg/l) } \\
\hline 1.0 & $40 d$ & $5 \pm 1.12 b$ & $30 \mathrm{e}$ & $15 \pm 1.22 \mathrm{~d}$ \\
\hline 1.2 & $50 \mathrm{e}$ & $12 \pm 1.45 c$ & $20 \mathrm{c}$ & $8 \pm 1.12 c$ \\
\hline 1.5 & $20 \mathrm{c}$ & $4 \pm 0.62 b$ & $48 f$ & $20 \pm 3.22 \mathrm{e}$ \\
\hline 2.2 & $48 \mathrm{e}$ & $10 \pm 2.22 \mathrm{c}$ & $10 b$ & $8 \pm 1.32 c$ \\
\hline 2.5 & $60 f$ & $16 \pm 2.12 d$ & $8 b$ & $8 \pm 1.62 c$ \\
\hline \multicolumn{5}{|l|}{ BAP+ NAA (mg/l) } \\
\hline $0.5+0.2$ & $30 \mathrm{c}, \mathrm{c}$ & $5 \pm 1.18 b, b$ & - & - \\
\hline $1.0+0.3$ & $40 \mathrm{~d}$ & $6 \pm 1.14 b, c$ & $34 d, b$ & $3 \pm 1.14 c, b$ \\
\hline $1.0+0.5$ & $30 \mathrm{~d}, \mathrm{~b}$ & $2 \pm 1.25 \mathrm{~b}, \mathrm{a}$ & - & - \\
\hline $1.5+0.1$ & - & - & $22 c, c$ & $6 \pm 1.15 \mathrm{~d}, \mathrm{c}$ \\
\hline $1.5+0.2$ & $68 \mathrm{e}, \mathrm{c}$ & $25 \pm 2.44 c, b$ & $68 c, d$ & $20 \pm 1.85 d, d$ \\
\hline $1.5+0.3$ & $30 \mathrm{e}, \mathrm{c}$ & $5 \pm 1.22 c, c$ & $12 \mathrm{c}, \mathrm{b}$ & $4 \pm 1.04 \mathrm{~d}, \mathrm{~b}$ \\
\hline $2.0+0.3$ & 74f,c & $30 \pm 1.14 d, c$ & 08b,b & $1 \pm 0.08 \mathrm{~b}, \mathrm{~b}$ \\
\hline $2.0+0.5$ & $52 \mathrm{f}, \mathrm{b}$ & $5 \pm 1.25 \mathrm{~d}, \mathrm{a}$ & - & - \\
\hline \multicolumn{5}{|l|}{ Kin+ NAA (mg/l) } \\
\hline $1.0+0.1$ & $40 \mathrm{~d}, \mathrm{~b}$ & $8 \pm 1.12 \mathrm{c}, \mathrm{b}$ & $40 \mathrm{e}, \mathrm{c}$ & $5 \pm 1.22 \mathrm{~d}, \mathrm{~b}$ \\
\hline $1.0+0.2$ & $60 \mathrm{~d}, \mathrm{c}$ & $15 \pm 1.25 \mathrm{c}, \mathrm{b}$ & $84 e, d$ & $35 \pm 1.12 d, d$ \\
\hline $1.5+0.2$ & $30 \mathrm{c}, \mathrm{c}$ & $3 \pm 1.12 \mathrm{a}, \mathrm{b}$ & 20d,d & $4 \pm 1.12 c, d$ \\
\hline $1.5+0.5$ & $35 c, c$ & $4 \pm 1.08 \mathrm{a}, \mathrm{b}$ & $10 \mathrm{~d}, \mathrm{~b}$ & $6 \pm 1.24 c, c$ \\
\hline $2.0+0.1$ & $42 \mathrm{e}, \mathrm{b}$ & $12 \pm 1.38 \mathrm{~d}, \mathrm{~b}$ & $20 \mathrm{c}, \mathrm{c}$ & $5 \pm 1.22 b, b$ \\
\hline $2.0+0.2$ & $38 \mathrm{e}, \mathrm{c}$ & $10 \pm 1.42 \mathrm{~d}, \mathrm{~b}$ & - & - \\
\hline $2.0+0.5$ & 70e,c & $20 \pm 1.32 d, b$ & - & - \\
\hline
\end{tabular}


Table 3. Effects of different concentrations and combinations of auxins in half-strength MS on adventitious root formation of in vitro raised sugarcane shoots. (Data were recorded after four weeks of culture)

\begin{tabular}{|c|c|c|c|}
\hline $\begin{array}{c}\text { Auxins } \\
(\mathrm{mg} / \mathrm{l})\end{array}$ & $\%$ of shoots rooted & $\begin{array}{c}\text { Average no. of roots } \\
\text { per shoot }( \pm \mathrm{SE})\end{array}$ & $\begin{array}{l}\text { Average length of } \\
\text { roots }(\mathrm{cm})( \pm \mathrm{SE})\end{array}$ \\
\hline \multicolumn{4}{|l|}{ IBA } \\
\hline 0.5 & - & - & - \\
\hline 1.0 & $20 \mathrm{~b}$ & $3 \pm 0.62 b$ & $3.0 \pm 0.40 b$ \\
\hline 1.5 & $30 \mathrm{c}$ & $4 \pm 0.32 \mathrm{a}$ & $3.5 \pm 0.70 \mathrm{a}$ \\
\hline 2.0 & - & - & - \\
\hline 2.5 & - & - & - \\
\hline \multicolumn{4}{|l|}{ NAA } \\
\hline 0.5 & - & - & - \\
\hline 1.0 & - & - & - \\
\hline 1.5 & $30 \mathrm{a}$ & $5 \pm 0.56 a$ & $4.2 \pm 0.72 \mathrm{a}$ \\
\hline 2.0 & $20 \mathrm{c}$ & $4 \pm 0.25 b$ & $3.4 \pm 0.44 c$ \\
\hline 2.5 & - & - & - \\
\hline \multicolumn{4}{|l|}{$\mathbf{I B A}+\mathbf{N A A}$} \\
\hline $0.5+0.5$ & - & - & - \\
\hline $1.0+0.5$ & $30 \mathrm{c}$ & $4 \pm 0.12 b$ & $3.0 \pm 0.20 \mathrm{~b}$ \\
\hline $1.5+0.5$ & $50 \mathrm{~d}$ & $7 \pm 0.52 c$ & $4.2 \pm 0.32 b$ \\
\hline $2.0+0.5$ & $10 \mathrm{~b}$ & $3 \pm 0.43 b$ & $3.0 \pm 0.52 b$ \\
\hline $2.5+0.5$ & - & - & - \\
\hline \multicolumn{4}{|l|}{$\mathbf{N A A}+\mathbf{I B A}$} \\
\hline $0.5+0.5$ & - & - & - \\
\hline $1.0+0.5$ & 98d & $12 \pm 0.36 c$ & $3.8 \pm 0.28 b$ \\
\hline $1.5+0.5$ & $70 \mathrm{c}$ & $10 \pm 0.25 b$ & $3.4 \pm 0.44 b$ \\
\hline $2.0+0.5$ & $50 \mathrm{~b}$ & $8 \pm 0.12 b$ & $3.2 \pm 0.32 b$ \\
\hline $2.5+0.5$ & - & - & - \\
\hline
\end{tabular}

$*_{-}=$no response. $* *$ Mean values followed by the same letter in the same column are not significantly different based on DMRT $(\mathrm{p}=0.05)$

Shoot tip and young folded leaf segments from the 10 Gy irradiated plants $\left(M_{1}\right.$ generation) were cultured onto MS medium supplemented with different concentrations and combinations of cytokinins (BAP, Kin and Zeatin) and auxin (NAA) for direct shoot regeneration. Young leaf segments produced the highest number of shoots when cultured on MS+1.0 mg/l Kin $+0.2 \mathrm{mg} / \mathrm{l} \mathrm{NAA}$ and about $84 \%$ explants proliferated shoots in this particular combination (Table 2). The mean number of shoots per culture was $35 \pm 1.12$ in the same medium (Plate 3). For indirect regeneration, $1.5 \mathrm{mg} / 1$ 2, 4-D with $10 \%$ coconut water $(\mathrm{CW})$ induced the highest percentage $(96 \%)$ of white friable calli from young leaf segments (Plate 1). MS+1.0 mg/l Zeatin $+0.2 \mathrm{mg} / \mathrm{l} \mathrm{NAA}$ along with $10 \% \mathrm{CW}$ was found best for indirect shoot regeneration (Plate 2). It was evident from the experiment that direct shoot regeneration was better than indirect regeneration. However, addition of $4 \%$ sucrose and $10 \%$ coconut water in the medium (MS $+1.0 \mathrm{mg} / \mathrm{l} \mathrm{Kin}+0.2 \mathrm{mg} / \mathrm{l} \mathrm{NAA}$ ) increased the number of shoot up to 45 per culture (Plate 4). Again, the highest shoot length $(7.2 \mathrm{~cm})$ was observed when urea $(100 \mathrm{mg} / \mathrm{l})$ was added to same medium (Plate 5). Finally, MS $+1.0 \mathrm{mg} / \mathrm{l} \mathrm{Kin}+0.2 \mathrm{mg} / \mathrm{l} \mathrm{NAA}+4 \%$ sucrose $+10 \% \mathrm{CW}+100 \mathrm{mg} / \mathrm{l}$ urea was selected for large scale shoot regeneration (Plate 6). On the other hand, Half-strength 
MS with $1.0 \mathrm{mg} / \mathrm{l} \mathrm{NAA}$ and $0.5 \mathrm{mg} / \mathrm{l} \mathrm{IBA}$ showed best root induction (Plate 7). Percentage of shoots rooted, average number of roots and average lengths of roots were $98,12 \pm 0.36$ and $3.8 \pm 0.28$ respectively on above mentioned medium (Table 3 ).
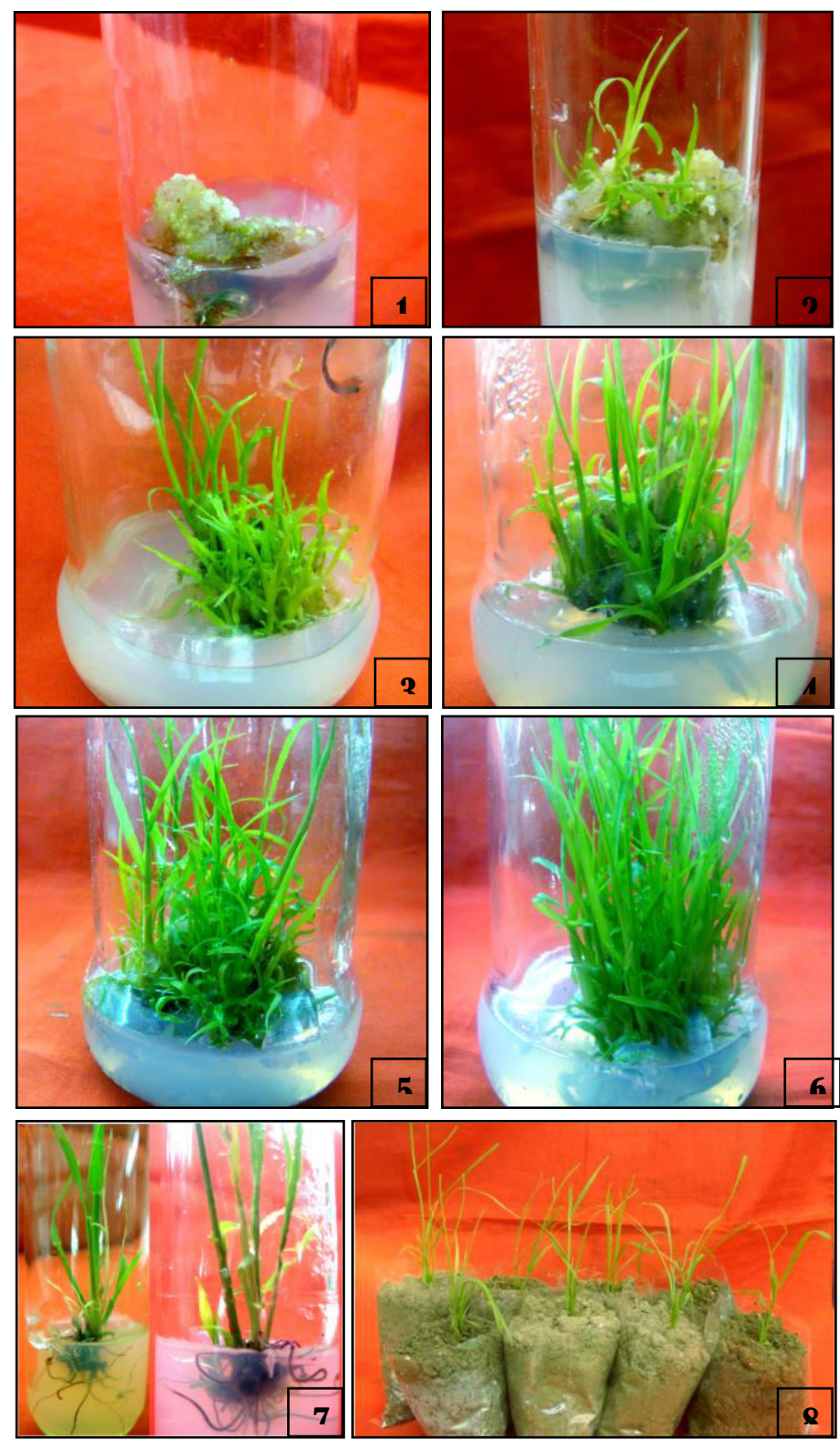

Plate.1-8. In vitro regeneration of Saccharum officinarum $\mathrm{L}$. var. Isd. 39. 1. Callus induction from folded leaf segment on MS $+1.5 \mathrm{mg} / \mathrm{l} \mathrm{2,4-D}+10 \% \mathrm{CW}$. 2. Shoot regeneration from callus on MS+ $1.0 \mathrm{mg} / \mathrm{l}$ Zeatin $+0.2 \mathrm{mg} / \mathrm{l}$ NAA along with $10 \% \mathrm{CW}$. 3. Multiple shoots regeneration from young leaf segment on MS+1.0 mg/l Kin + 0.2 mg/l NAA. 4. Positive effect of $4 \%$ sucrose and $10 \% \mathrm{CW}$ on shoot development. 5. Elongated shoots on MS+ $1.0 \mathrm{mg} / \mathrm{l} \mathrm{Kin}+0.2 \mathrm{mg} / \mathrm{l} \mathrm{NAA}+100 \mathrm{mg} / \mathrm{l}$ urea. 6 . Healthy and elongated multiple shoots on MS $+1.0 \mathrm{mg} / \mathrm{l} \mathrm{Kin}+0.2 \mathrm{mg} / \mathrm{l} \mathrm{NAA}+4 \%$ sucrose $+10 \% \mathrm{CW}+100$ $\mathrm{mg} / \mathrm{l}$ urea. 7. Root induction on half -strength MS $+1.0 \mathrm{mg} / \mathrm{l} \mathrm{NAA}+0.5 \mathrm{mg} / \mathrm{l} \mathrm{IBA} .8$ Acclimatized plantlets in poly bag soil 
There are many reports on regeneration of shoots from shoot tip explant of mature plants. Pathak et al. (2009), Singh et al. (2001) reported that optimum number of shoot was produced in MS medium supplemented with BAP, Kin and NAA $(0.5 \mathrm{mg} / \mathrm{l} \mathrm{each})$. Similar results were obtained by Biradar et al. (2009) with BAP (2.0 mg/l), Dhumale et al. (1994) with BAP at the rate of $3.0 \mathrm{mg} / 1$ and NAA with $1 \mathrm{mg} / 1$ in case of sugarcane which supported the present findings. Ali et al. (2008) observed the best shoot regeneration from shoot tip explants in MS medium with $1.5 \mathrm{mg} / \mathrm{l}$ BAP. Roy and Kabir (2007) reported best shoot proliferation in MS media with $1.5 \mathrm{mg} / \mathrm{l} \mathrm{BA}$ from shoot tip explants. For acclimatization and plant establishment under the natural conditions, the well-rooted plantlets were transferred to poly bag containing soil and compost in the ratio of 2:1 (Plate 8). During hardening $70 \%$ plantlets survived and these were subsequently transferred to experimental field.

\section{REFERENCES}

Ahloowalia, B.S. and Maluszynski, M.2001. Induced mutations: A new paradigm in plant breeding. Euphytica.118:167-73.

Ali, A., Nazi, S., Siddiqui, F.A. and Iqbal, A.J. 2008. An efficient protocol for large scale production of sugarcane through micropropagation. Pak. J. Bot. 40(1): 139-149.

Anonymous, 1953. Annual Report of Communication Incharge of the Experimental Station. Hawaiian Sugar Planters Association. p. 44.

Bermejo, A., Pardo, J. and Cano, A. 2011. Influence of gamma irradiation on seedless citrus production, pollen germination and fruit quality. Food Nutr Sci. 2: 169-80.

Biradar, S.D.P., Patil, V.C., Patil, S.S. and Kambar, N.S. 2009. In vitro plant regeneration using shoot tip culture in commercial cultivar of sugarcane. Karnataka J. Agric. Sci. 22(1): 21-24.

Brunner, H.1995. Radiation induced mutation for plant selection, 4PPL Radiat. pp. 589-594.

Dhumale, E.D., Ingloe, G.L. and Durge, D.V. 1994. In vitro rgeneration of sugarcane by tissue culture. Ann. Pl. Phys. 8: 192-194.

Federer, W.T. 1963. Experimental design, Macmillan Press, New York. pp. 214-285.

Feldmenn, P., Sapotille, J., Gredoire, P. and Rott, P.1994. Micropropagation of sugarcane. In:Teisson, $C$. (ed.). In vitro culture of tropical plants. pp.15-17. France.

Khan, I.A., Khatri, A., Ahmad, M., Siddiqui, K.A., Dahar, N.A., Khanzada, M.H.and Nizamani, G.S. 1997. Genetic superiority of exotic clones over indigenous clones for quantitative and qualitative traits. The Nucleus. 34: 153-156.

Khan, I.A., Dahot, M.U. and Khatri, A. 2007. Study of genetic variability in sugarcane induced through mutation breeding. Pak. J. Bot. 39(5): 1489-1501.

Khan, I.A., Dahot, M.U., Seema, N., Asmeen, S., Bibi ,S., Raza, G., Khatri, A. and Naqvi, M.H. 2009. Direct regeneration of sugarcane plantlets: a tool to unravel genetic heterogeneity. Pak. J. Bot. 41(2): 791-814.

Khan, I.A., Khatri, A., Siddiqui, M.A., Nizamani, G.S. and Raza, S. 2004. Performance of promising sugarcane clone for yield and quality traits in different ecological zones of Sindh. Pak. J.Bot. 36(1): 83-92.

Khan, S. and Wani, M.M.R. 2005.Genetic variability and correlations studies in chickpea mutants. J. Cytol. Genet. 6(2): 155-160.

Krisnamurthi, M. and Tlaskal, J. 1974. Fiji disease resistant, Saccharum officinarum L. var. Pindar subclone from tissue culture. Proc. ISSCT. 15:130-137.

Kwon-Ndung, E.H. and Ifenkwe, O.P. 2000. Mutation studies on sugarcane using gamma irradiation. Sugar Tech. 2: (1\&2): 29-35. 
Lal, N. and Krishna, R.1994. Sugarcane and its problems: Tissue culture for pure and disease free seed production in sugarcane. Indian sugar. 44: 847-848.

Lee, T.S.G. 1987. Micropropagation of sugarcane (Saccharum spp.). Plant Cell Tissue Org. Cult.10: 47-55.

Lorenzo, J.C., Ojeda, E., Espinosa, A. and Borroto, C. 2001. Field performance of temporary immersion bioreactor derived sugarcane plantys. In vitro cell Dev. Biol., Plant.37: 803-806.

Nwachukwu, E.C., Ene, L.S.O., Mbanaso, E.N.A. and Ago, F.M.O. 1990. Increasing variability in yams by induction of mutation using gamma rays.Annual Report. National Root Crops Research Institute Amidic, Omaha. Nigeria. pp. 123-145.

Pathak, S., Lal, M., Tiwari, A.K .and Sharma, M.L. 2009. Effect of growth regulators on in vitro multiplication and rooting of shoot cultures in sugarcane. Sugar Tech. 11(1):86-88.

Pavadai, P., Girija, M. and Dhanavel, D.M. 2009. Effectiveness and efficiency and biochemical content of physical and chemical mutagens in soybean (Glycine max (L.) Merr.). Journal of Phytology.1(6): 444- 447.

Pepol, P. and Pepo, P. 1989. Preliminary experiment on inducing soybean mutants by fast neutron seed irradiation. Soybean Abstracts. 12(5): 4-7.

Prabhakar, L.V.1985.Studies on induced mutagenesis in Sesamum indicum L. M.Sc. (Ag.) Thesis, Tamil Nadu Agricultural University, Coimbatore, India.

Predieri, S. 2001. Mutation induction and tissue culture in improving fruits. Plant Cell Tiss Org. 64: $185-210$.

Rehman, S., Khan, G.S. and Khan, I. 1992. Coordinated uniform national varietal trial on sugarcane. Pak. J. Agri. Res. 13: 136-140.

Roy, P.K and Kabir, M.H. 2007. In vitro mass propagation of sugarcane (Saccharum officinarum L.) var. Isd 32 through shoot tips and folded leaves culture. Biotechnology. 6(4):588-592.

Singh, B., Yadav, G.C. and Lal, M. 2001. An efficient protocol for micropropagation of sugarcane using shoot tip explants. Pak. J. Bot. 3(3): 113-116.

Yasmin, S., Khan, I.A, Khatri, A., Seema, N., Siddiqui, M.A. and Bibi, S. 2011. Plant regeneration from irradiated embryogenic callus of sugarcane. Pak. J. Bot. 43(5): 2423-2426. 